\title{
Il. JAIIRESBERICIITE.
}

\section{Dio Cassius.}

(S. Philol. XI, P. 139.)

IV. Die zeit vom ende des dritten makedonischen krieges bis zum ausbruche des bürgerkrieges zwischen

Caesar und Pompejus.

1. R. Wilmuns, De fontibus et auctoritate Dionis Cassii. Berolini. 1835. $8^{0} .2$ bl. $46 \mathrm{p}$.

2. F. Eyssenhardl, Bemerkungen zu der frage über die glauhwürdigkeit von Caesars commentarien. Jalirbücler für classiscle philologie. Bd. 85. 1862, p. 755-764.

3. Max Grasshof, De fontibus et auctoritate Diouis Cassii Cocceiani. Bonnae. 1867. $8^{0} .1$ bl. 44 p.

4. F. W. Laucr, De scriptoribus belli Mitbridatici tertii. Programm des gymnasiums zu Wetzlar. 1871. $4^{0} .1-16 \mathrm{p}$.

5. E. Klebs, De scriptoribus aetatis Sullanae. Berolini. 1876. 80.1 bl. $64 \mathrm{p}$.

6. H. Dübi, Die jüngeren quellen der Catilinarischen verschwörung. Jahrbücher für classische philologie. Bd. 113. 1876, p. $851-879$.

7. Schlicplacke, Ueber die griechischen quellen zur Catilinarischen verschworung. Programm der realschule erster ordunng zu Goslar. 1877. 40. 1-37 p.

8. G. Thourel, De Cicerone, Asinio Pollione, C. Oppio rerum Caesariauarum scriptoribus. Lips. 1878. $8^{0}$. (Auch in: Leipziger studien zur classischen philologie. Bd. I. p. 303-360).

9. J. Besser, De coniuratione Catilinaria. Neostadii ad Orlam. (Leipziger dissertation). 1880. $8^{\prime \prime}$. 
Bis zum dritten mithridatischen kriege.

Die wichtigen fragmente des Dio Cassius, welche die geschichte der zerstörung Kartlago's, des vierten makedonischen und des achaeischen krieges behandeln, sind von der quellenforschung bisher übergangen worden, und auch die über Dio's darstellung des numantinischen krieges und der gracchischen unrulıeu geführten untersuchungen haben den vorhandenen stoff nur zum geringen theile ausgebeutet. Erst in der sullanischen zeit stehen wir wieder auf festerem bodeu.

Auf die benutzung der verlorenen bücher des Iivius durch Dio hat nach Perizonius (Animadversiones historicae. Altenb. 1771, p. 185 etc.) zuerst Wilmaus (nr. 1, p. 14-19) aufmerksam gemacht, indem er eine reilie von stellen des Obsequens und Florus mit den fragmenten des Dio zusammenstellte. Bei der darstellung der letzten zeit der republik, namentlich des krieges zwischen Caesar und Pompejus hat Dio nach Wilmans' ansicht seinen bisherigen führer verlassen, um sich au quellen, die auf der seite des Caesar und Augustus standen, anzuschliesseu. Für die benutzung des $\mathrm{Li}$ vius durch Dio für die epoche der bürgerkriege haben sich auch Iagus (Plutarchus Livii studiosus. Helsingfors. 1848, p. 10) und Schiern (De originibus et migrationibus Cimbrortm. Hanniae. 1842, p. 80) ausgesprochen.

Die von Hoffmann (De Viriathi Nomantinorumque bello. Gryph. 1865 , p. 10) geäusserte vermuthung, dass Dio für die kriege mit Viriathus und Numantia dem Livius adhibitis tamen aliis quoque auctoribus gefolgt sei, beruht auf der früher besprochenen combination von H. Nissen, die auch van Geer (De fontibus Platarchi in vitis Gracchorum. Iugd. Bat. 1878, p. 83) dazu veranlasst hat, Dio s nachrichten uber die graccbischen unruhen auf Isivius zurückzuführen und damit seiner lypothese von der aushilfsweisen benutzung des Livius in den plutarcbischen biographieen der Gracchen eine neue stütze zu geben. Wenn lier allerdings auch die quellen sehr spärlich fliessen, so liätte doch wenigstens auf die nahe verwandtschaft von Dio fr. 84,2 mit Jul. Obsequens 28 (87) und mit Augustinus de civ dei III, 11 hingewiesen werden kïnnen.

Eine sehr ungünstige beurtheilung erfährt dieser abschnitt des Dio durch Nitzsch (Oie Gracchen. Berlin 1847, p. 447), der in jenen fragmenten manche ,unangenehme übertreibungen" und wenig verlässiges detail findet, über Dio's muthmassliche quellen sich aber nicht weiter äussert.

Ueber die zeit vom jahre 113-80 erhalten wir durch die mit grossem fleisse geführten untersuchungen von Klebs (nr. 5, p. 41-43) erwünschte auskunft. Er hat die von Wilmans gesammelten parallelen zwischen den fragmenten des Iivius und Dio um 
eine heträchtliche anzahl vermehrt und die ansschliessliche benntzung des Livius für die zeit des Sulla angenommen, da er es für ausgemacht lält, dass Dio die gleichzeitige beiziehung mehrerer geschichtsqellen grundsätzlich vermieden habe.

Wir brauchen nach unseren früheren erorterungen diese lypothese nicht erst hier ausdrücklich zu widerlegen und darauf hinzuweisen, dass es gerade erst die künftige anfgabe der qnellenforschung sein wird, wie für die übrigen historiker des alterthums, so auch speciell für Dio die von ilm befolgte methode der quellenbenutzung festzustellen. Für den von Klebs behandelten abschnitt aber ist von hoher wichtigkeit die bisher übersehene äusserst nahe verwandtschaft zwischen dem Bellum Iugurthinum des Sallust und folgenden stellen des Dio Cassius:

Dio fr. 89,1
Sall. lugurth. 62,5

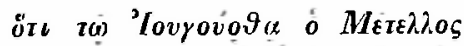

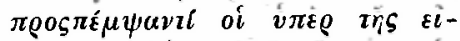

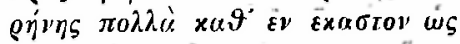

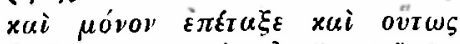

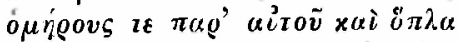

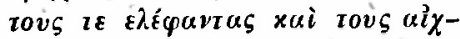

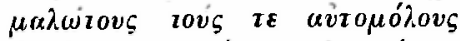

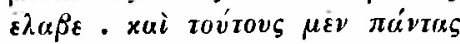

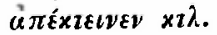

Dio fr. 89,4

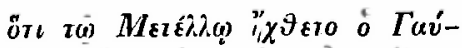

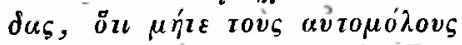

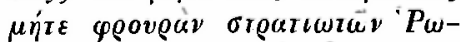

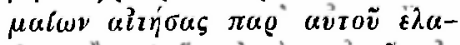

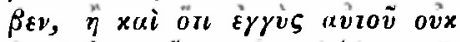

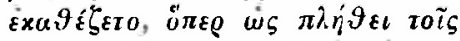

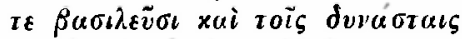

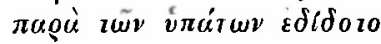

lugurthae imperat argenti poudo ducenta milia, elephantos omnis et armorum aliquantum . quae postquam sine mora facta sunt, iubet omnis perfugas vinctos adduci e eorum magna pars adducti, pauci ... abierunt.

Vgl. ausserdem Dio fr. 89,2 mit Sall. lug. 63. Dio fr. 89,3 mit Sall. lug 65. 64, 4.

Wie Gauda hätte dazı kommen sollen, die römischen überläufer sich von Metellus auszubitten ist unbegreiflich und kann keinesfalls in der von Dio benutzten quelle gestanden haben. Der zusatz der ersten stelle, dass Metellus ausser den überläufern auch geisseln und die gefangenen sich habe ausliefern und die überläufer habe hinrichten lassen, wäre ohne schwierigkeit auf eigene combination des Dio zurückzuführen. Auf desertion stand allezeit todes- 
strafe (Liv. XXIV, 20. 45. XXX, 43. ep. I. IL. Paul. Dig. 48, 19, 38. Mart. ib. 8, 3). Trotzdem halten wir es nicht für unmöglich, dass Dio hier den Sallust auf indirektem wege, am wahrscheinlichsten durch die vermittelung des Livius benutzt hat. Dafür scheint erstlich die nahe rerwandtschaft des bei Orosius stehenden auszugs aus Livius mit einzelnen capiteln des Sallustianischen lugurtha zu sprechen, ferner der umstand, dass auch bei Orosius V, 15 lugurtha geiseln stellen muss.

Die auf den ersten mithridatischen krieg bezigglichen fragmente des Dio hatte vor Klebs schon Jordan (De fontibus Appiani in bellis Mithridaticis enarrandis. Gott. 1872, P. 35. 50 etc.) mit Livius in verbindung zu bringen gesucht.

\section{Die jahre 69-66 (Lib. XXXVI c. 1-44).}

Einem glücklichen zufall haben wir es zu verdanken, dass wir wenigstens mit einiger wahrscheinlichkeit über den ursprung der von Dio Cassius über den ersten theil des dritten mithridatischen krieges und den feldzug des Pompejus gegen die seeräuber gemachten angaben zu urtheilen vermögen. Waren uns nicht einige wenige an sich ziemlich unbedeutende fragmente der historien des Sallust erhalten, so hatte gewiss auch hier Dio Cassius als epitomator des Livius gelten müssen, mit den er wenigstens für den grösseren theil dieser periode nichts zu thun zu haben scheint. Es ist das verdienst von Wilmans (nr. 1 p. 6-14), zuerst auf die wahrscheinlichkeit einer benutzung der histori en des sallust durch Dio hingewiesen zu haben. Seine beweisführung ist von $\mathbf{H}$. Peter (Die quellen Plutarchs in den biographieen der Römer p. 108) gebilligt, von Grasshof (nr. 3 p. 3-11) fast unverändert aufgenommen worden. Erst Lauer (nr. 4 p. 2-3) hat wieder die sehr schwierige frage nach dem verhälnisse der plutarchiscben biographie des Lucullus zu Dio und Livius wieder selbständig erörtert und durch die heranziehung von zwei neuen fragmenten des Sallustius die veumuthung von Wilmans bestätigt, wahrend fast gleichzeitig Jordan (Ine fontibus Appiani in bellis Mithridaticis enarrandis. Gotting. 1872, J. $74-80$ ) dieselbe zu widerlegen und Livius als den hauptgewährsmann des Dio Cassius zu erweisen suchte. Die bisher auf Sillust zurückgefülırten stellen des Dio sind folgende:

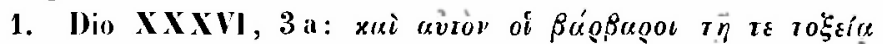

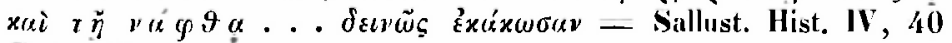
Gerl. : Niphtis.

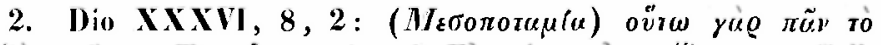

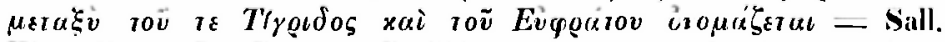
Hist. IV, 52 I). (Kr. 11. G. 55): Tigrim et Fuphratem uno fonle 
manare in Armenia ... quae tamen terra, quae ab ipsis ambitur, Mesopotamia dicitur.

3. Dio XXXVI, 17, 3: oi $\gamma \varepsilon O v \alpha \lambda \varepsilon g \ell \varepsilon \iota 0 \iota, \mu \alpha 9 o ́ v t \varepsilon \varsigma$

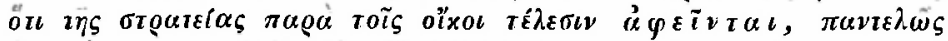
$\ddot{\imath} \pi \varepsilon \chi \dot{\omega} \varrho \eta \sigma v=$ Sall. Hist. V, 10 D. (Kr. 14. G. 2): Legiones Valerianae comperto lege Gubinia Billyniam et Pontum consuli datam, sese missos esse.

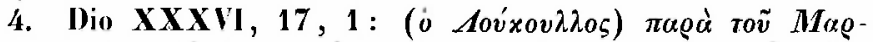

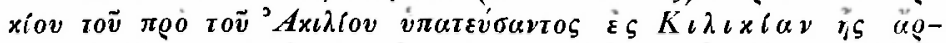

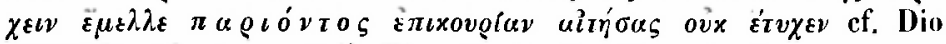
XXXVI, 16, 4. = Sall. Hist. V, 11 D. (Kr. 12. G. 1): at Lucullus audito O. Marcium Regem pro consule per Lycaoniam cum tribus legionibus in Ciliciam tendere.

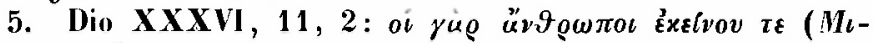

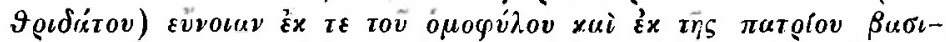

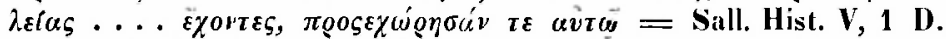
(Kr. 1. G. IV, 7!) : adeo illis ingenita est sanctitas regii nominis.

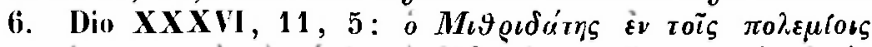

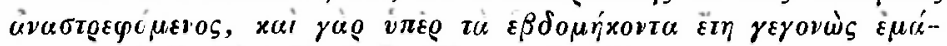
$\chi \varepsilon \tau o=$ Sall. Hist. V, 3 D. (Kr. 4. G. 10): peractis septuaginta annis armatus equm insilire.

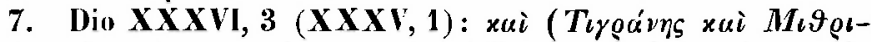

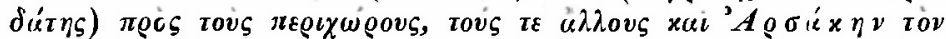

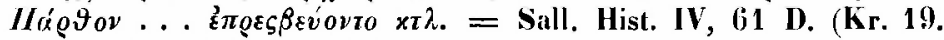
G. p. 155): Epistula Mithridatis. Rex Mitluridates regi Arsaci salutem etc.

Bisher seltsamerweise übersehen, aber sicher aus Sallust geflossen ist ferner der satz:

8. Dio XXXVI , 19, 2: Máoxios $\delta \varepsilon$ Movxov $\lambda \lambda(\omega) \mu \varepsilon \nu$ oùx

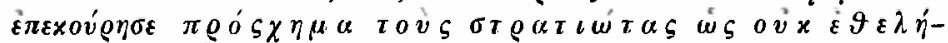

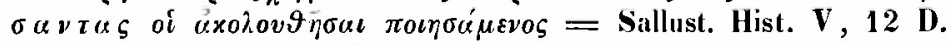
(Kr. 13. G, 11): Set ubi ille militum voluntatem causatus.

Offenbar kommt niclıt allen angefülırten stellen die gleiche beweiskraft zu und können wir als entscheidend eigentlich nur die vierte und die beiden letzten paralleien ansehen. Den ausschlag giebt der dem Dio und Sallust gemeinsame fehler, dass Mithridates nach der schlacht bei Tigranocerta (6. oct. 685) an den entweder zu ende des jalıres 684 oder zu anfang des jalıres 685 gestorbenen Partlerkönig Arsakes eine gesandtschaft schickt, die vielmehr dessen nachfolger Phraates gegolten hat. Das bei Plutarch Luc. c. 33 stehende fragment des Sallustius über den soldatenaufstand im lager des Lucullus lat Grasshof ebenfalls mit Dio XXXVI, 18 in bezielung gesetzt, dabei aber ïbersehen, dass Sallust an jener stelle des Plutarch nur als gewährsmann für die bei Cyzicus und Amisum unter den römischen legionen ausgebrochenen unruhen genannt wird, während der späteren meutereien auch von anderen 
und namentlich von I.ivius (ep. I. XCVIII Lucullum . . sedilio mililum lenuil qui sequi nolebanl. id est legiones Valerianae, quae implela a se stipendia dicentes Lacullum reliquemunt) erwähnung geschieht.

Von Plutarch und Appian, die für diese periode walırscheinlich zumeist dem Idivius gefolgt sind, weicht Mio mehrfach in sehr aufallender weise ab, vor allem aber darin, dass er von einem zweiten zwischen der schlacht von Tigranocerta und der einnahme von Nisibis erfochtenen siege des Incullus keine kunde hat. Ia aber auch Livius (bei Plut. Isuc. c. 31) von der sogenamnten schlacht bei Artaxata berichtet hat, so ist schon damit Jordans behauptung widerlegt, wornach Dio's anklänge an Sallust aus einer benutzung des Livius zu erklären seien, der wieder seiuerseits den Sallustius zu grunde gelegt haben soll. Wollen wir nicht allzu skeptisch verfahren, so müssen wir vielmelır an Sallust als an der hauptquelle des Dio für die geschichte der unternelimungen des Incullus festhalten. Eine gleichzeitige benutzung des Iivius ist damit nicht ausgeschlossen, vielmelır durch die folgenden stellen sehr nalie gelegt:

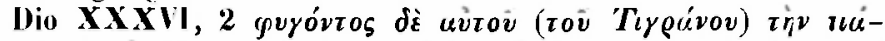

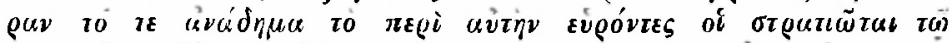

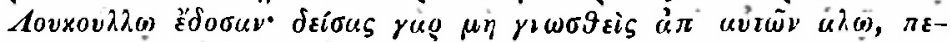

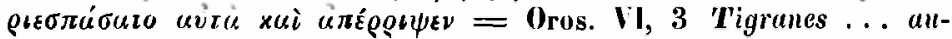
figit, diademale el liara ne agnoscerelur abjectis. Von Plutarch Iuc. c. 28 wird eine ganz verschiedene darstellung dieses vorganges gegebell.

Dass auch die erzählung der gleichzeitigen ereignisse in der hauptstadt von Dio aus Sallust geschöpft ist, folgert Wilmans aus der allerdings ganz frappanten übereinstimmung derselben mit einigen stellen des Cicero-conmentares des Asconius Pedianus, der sich iber seine quellen mit den worten ausspricht: neque apud Sallustium, neque apud Livium, neque apud Feneslellam ullius alterius ab eo latue legis montio (V,2 p. 66 ed. Orelli). S'cheint es auch sehr gewagt, mit Wilmans von diesem citate auf die vorwi egende bcuutzung des Sallustius durch Asconius zu schliessen (ur. 1, p. 13: ila aperte innuil se in cognoscendis rebus hoc lempore gestis imprimis Salluslium consuluisse), so glaubeu wir doch nach einer anderen seite ziemlich bestimmte hinweise auf die quelle des Dio zu finden. Eine genauere vergleicbung von Plutarch's und Dio's bericbt über den seeräuberkrieg des Pompejus lïsst nämlich eine sel:r nahe verwandtschaft beider darstellungen erkennen, die sich namentlich an folgenden stellen ausspricht:

$$
\text { Dio } \mathbf{X X X} \text { VI, 30, } 3
$$

Plut. Pomp. c. 25

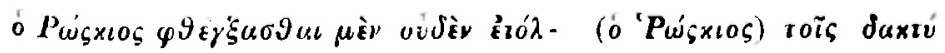

Philologus. XLI, bd. 1. 


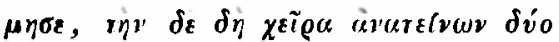

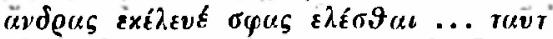

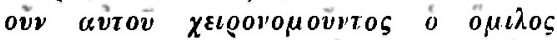

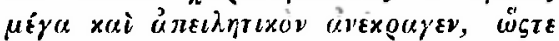

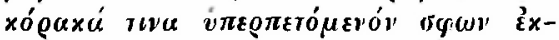

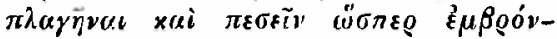
тทrov.

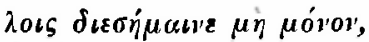

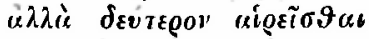

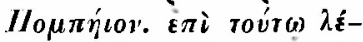

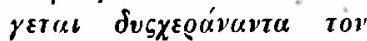

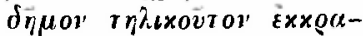

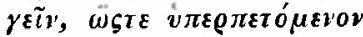

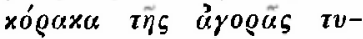

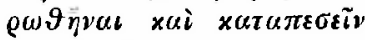

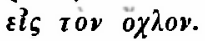

Plut. Pomp. c. 28

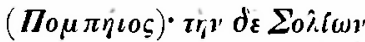

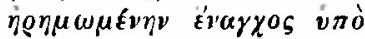

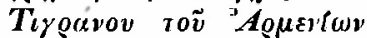
$\beta a \sigma i \lambda \varepsilon \omega \varsigma$ Q⿱⿲㇒丨丶㇒⿴囗⿱一一

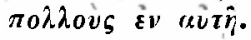

Nun hat sich zwar H. Peter (a. a. o. p. 114) iiber die quelle des Plutarch, als welche er die historien des Sallust bezeichnet, mur ziogernd ausgesprochen. Allein angesichts dessen, dass in den dem seeräuberkriege vorausgehenden abschnitten der biographic des Pompejus eine ganze reihe ron sallustianischen fragmenten sich wiederfindet, andererseits der von Dio nnd Plutarch iiberlieferten version die bei den epitomatoren des Livius erhaltenen nachrichten in mehreren punkten widersprechen, scheint zu irgend welchem bedenken ein grund niclit gegeben. (Ganz im tone der moralistischen declamationen des Sallustius ist namentlich die folgende stelle des

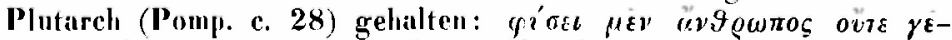

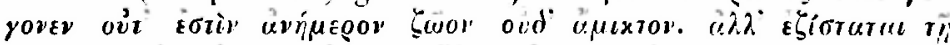

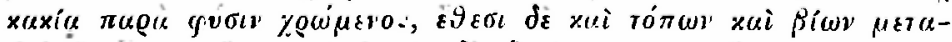

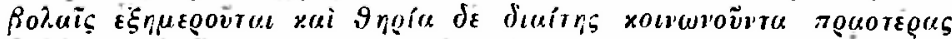

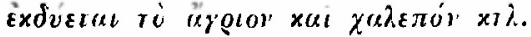

Wir können also mit ziemlicher sicherheit vermittels der hesprochenen aluschnitte des Dio Cassius und Plutarchus wenigstens einen theil des wichtigen sallustianisclien geschichtswerkes reconstruiren.

\section{Die feldziige des Pompejus in Asien 66-62 (Lib. XXXVI, $45-$ XXXVII, 22).}

Beziiglich dieser periode kïnnen wir uns kurz fassen, da hier nur Grasshof's (nr. 3, p. 11-13) und Jordan's (De fontilus Appiani in bell. Mithrid. enarrandis p. 85-107) untersnchungen in frage kommen, die beide in Livius die hauptquelle des Dio erkennen; Jordan allerdings liiss, um die durchgangige abhiangigkeit 
der appianischen darstellung der mithridatischen kriege von Iivius aufrecht zu erlalten, den Dio an den von Appian abweichenden stellen eine von ilım nicht näher charakterisirte secundärguelle heranzielıen. - Von Grasshof sind die angaben der livianischen epitomatoren nur zum theile ausgenutzt worden; namentlich ware hier Florus melır zu berücksiclitigen und die frage ernstlich zu erortern gewesen, ob denn wirklicl, wie H. Peter (a. a. o. p. $114-$ 117), annimmt, Plutarch's schilderung der asiatischen feldzüge des Pompejus in ilırem ganzen umfange anf Theophanes zurückzufülıren ist. Auf die nahe verwandtschaft von Plut. Pomp. 32, 6 mit Eutrop VI, 12 und Orosius VI, 4, von Plut. Pomp. 33, 2 mit Eutrop VI, 13 und Dio XXXVI, 52, von Plut. Pomp. 36, 4 mit Florus I, 40, 28 aufmerksam machend, glaube ich besonders an der folgenden stclle einen ganz bestimmten hinweis anf die wenn anch nur subsidiare verwendung des Iivius inch Plutarch zu finden:

\section{Dio XXXVI, 4!), 7 Flor. I, 40, 23 Plut. Pomp. 32, 5}

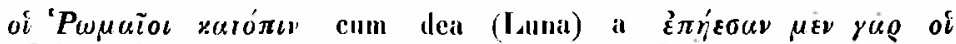

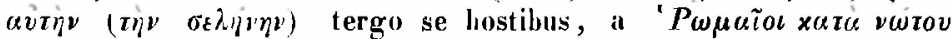

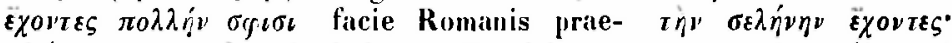

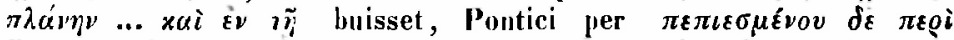

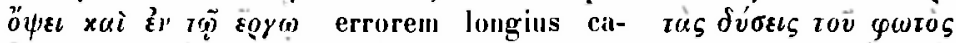

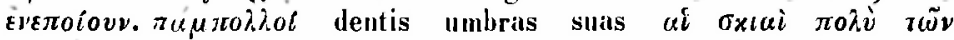

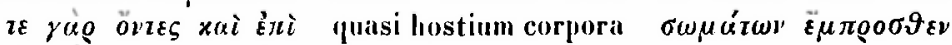

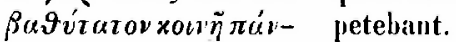

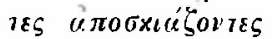

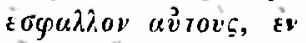
(i) $\gamma \varepsilon$ oข้

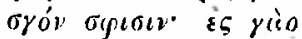
Iò xqvov of $\beta$ rio $\beta$ uool,

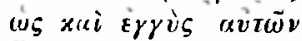

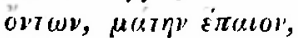

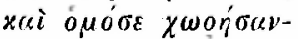

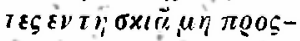

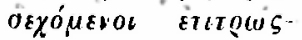

\section{Oros. 1I, 4}

Iuná tunc orta a lergo Romanis erat. Regii longitudinem umbrarum proximitatem hostium rati cuncta in irritum tela fuderunt.

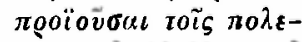

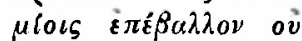

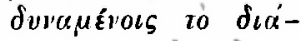

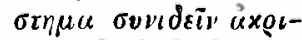

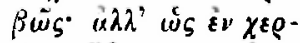

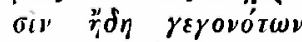
lovg vorois ugevtes

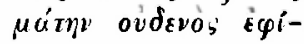
xorzo.

xovto.

Während Florus eine sinnlose parodie der livianischen darstellung giebt, hat Plutarch wohl nur aus verschen den monduntergang für den aufgang gesetat.

Was iibrigens die frige nach der quelle Dio's betrilft, so darf seine ablïugigkeit von Livius als ziemlich gesichert gelten und kann uns namentlich Dio's erzählung von dem tode des Mitbridates zu einem zweifel an der richtigkeit jencr annahme nicht veranlassen. (Erisshof (11r. 3, ए. 12) bemerkt hicriiher: Ea de re Dio 
ipse nescivisse videtur, quid dical; dum enim XXXV11, $10 \mathrm{M}$ thridaten sibi ipsum mortem dedisse dicit, XXXVII, 12 regem Pharmacis inssu interfectum esse, et XXXVII. 13 a mililibus eum occisum esse trudil etc. Dieser widerspruch ist aber doch nur ein

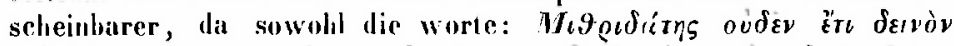

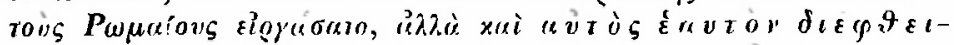

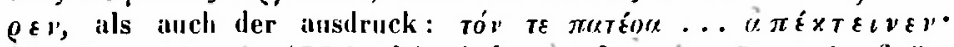

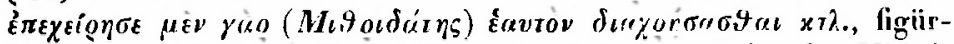
lich gebraucht sind. Nach Jivius aber hat der mörder des Mithridates nicht dessen treugebliebenen anhängern, sondern der von Pharnaces gegen ihn ansgesandten truppe angehört (Oros. VI, 5 Gallum quendam mililom iam fraclo muro discurrentem invilavil ancl. de vir. ill. 76 immiss am percussorem Gallum); mit ihm stimmt Dio, nicht aber Appian M Mo 90.111 überein (vgl. Jordan a. a. 0. p. 101-103). - Auf die von Vollgraff (Greek writers of Romin history. Leyden 1880) geäusserte vermuthung, dass könig Juba's Archaeologia Romana eine hauptguelle Plutarch's und Appian s für die geschichte des letzten jahrhumderts der römischen republick gew esen, hier niher einzugeben düren wir uns um so elher versagen, als Vollgraff seine theorie, dass jene beiden autoren nothwendig aus griechischer quelle geschöpft haben müssten, auf Dio Cassius - ich weiss nicht aus welchem grunde - nicht ausgedehnt, sondern für ihn die direkte benutzung des Livius mehrfach zugegeben hat (a. a. o. p. 6it. 105 etc.).

\section{Die catilinarische verschwörung (1. XXXVII, 29-41).}

Ueber den werth und die glaubwürdigkeit von Dio's darstellung der catilinarischen rerschnörung gehen die meinungen so weit inseinander, dass es mothwendig ist, der erörterung der quellenfrage eine chronologische zusaumnenstellung der bisher geäusserten vermuthungen voriuszuschicken. Dic ansgebreitetste belesenheit wird dem Dio von Wilmans (nr. 1, p. 27-31) zugeschrieben, der von ihm nicht nur Sallust's Catilina, sowie die reden und das buch megi inazeios des Cicero, sondern anch Livius, Plutarch und Diodor benutzt sein lässt. Während auch Lagus (Plulurchus vilue Ciceronis scriplor. Helsingfors. 1846 II. p. 87.133 etc.), der Dio's bericht in seiner trefflichen abhandlung leider nur ganz gelegentlich behandelt, in ('icero's memoiren eine hauptiguelle des Dio und des plutarchischen Cirero (vgl. Ilieren, Comm. IV de font. et auct. vit. parall. Plnt. Vermischite histor. schr. Gott. 1821, III, p. 23, 417) zu finden glauble, hat H. Peter (Die quellen Plutarchs p. $130 \mathrm{ff}$.) auf die jener annalime entgegenstchenden bedenken hingewiesen und Livius als den gemeinsamen gewährsmann Dio's und Plutareh's bezeichnet; ihm haben sich Girasshof (nr. 3 p. 13-17) und John (Die entslehungsgeschiche der catilinarischen versehwio- 
rung. Jilhrbücher fïr classische plitologie. Supplementband. 8. $1875-1876$. p, 722. 756 etc.) ohne anführung neuer beweismomeute vollstindig angeschlossen. Wiedemann aber (Ueber Siallust's Catilin. c. 27, 3 - c. 28, 3. Philologus XXII, 1. 498) kommt wieder anf das von Wilmaus ausgesprochene nrtheil zurück und sucht einen grösseren theil von Dio's darstellung direkt auf Sillust, Appian und Plutiıch zurückzuführen. Noch weiter geht I)übi (nr. (j p. 877), nach dessen ansicht dem Dio das ganze material zur geschichte der catilinarischen verschwörung, also ausser den eben genannten namentlich Livius vorgelegen hat; diese sämutlichen berichte aber habe Dio in ausserst füchtiger weise und mit zufügung eigener erfudungen und combinitionen verirbeitet. Von Schliephlicke (nr. 7 p. 31 - 37) wird die zall] der von I)io benutzten quellen wieder auf "jallust, Livius und Appian besclıränkt und Thouret (nr. 8) hat nach dem vorgange von Weizsäcker (Ciceros Hypomnema und Plutirch. Jalırbl. f. philol. bd. 111. 1875. p. -117. 428) abermals die direkte benutzung von Cicero's memoiren Wahrscheinlich zu machen gesucht. Ausser Cicero haben nacl Bessers (ur. 9) wenig überzeugender beweisführung iuch Livius und Sallust dem Dio als vorlage gedient.

Als der einzige feste punkt in dem clias widerstreiteuder ausichten ist dic aligemeine ancrkemung der nahen verwandschaft zwischen den angaben des Dio und des plutarchischen Cicero zu bezeichnen. Und zwar ist dieselbe von der art, dass wenigstens in der überwiegenden mehrzahl der fälle die ron Wilmans, Dübi und Wiedeminn beliebte anmahme einer benutzung des Plutarch durch Dio von vornelıerein ausgeschlossen wird. Ist auch IVio's darstellung, wie immer, eine flüchtige und verwaschene, so wird doch di und dort erst durch sie Plutarch's erzählung in das rechte licht gesetat und während Dio im ganzen die thatsichen in derselben reihenfolge wie Plutarch und abweichend von Sillust anfführt, erwähnt er manche einzelheiten, die von Plutarch übergangen worden sind. - Fur die charakterisirung der gemeinsimen quelle des Dio und Plutirch sind von höchster wichtigkeit die von Iagus und Weizsicker gesimmelten stellen aus einzelnen redeu des $\mathrm{Ci}$ cero, die zum theile wort fur wort den angaben jener beiden historiker entsprechen. So unwihrscheinlich eine direkte benutzung der ciceronianiscben reden durch Dio, wie sie Wilmans angenommen, erscheinen muss, so leicht liisst sich jene übereinstimmung zwischen Plutarch und Dio aus der ahhängigkeil beider autoren

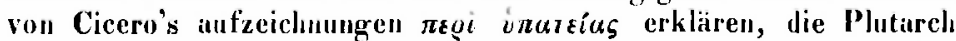
nach seinen eigenen worten mehrfach eingesehen hat (Cratss. 13. Cines. 8) und die auch dem IDio Cassius genau bekann waren (Dio

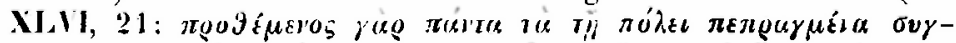

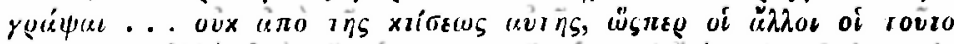

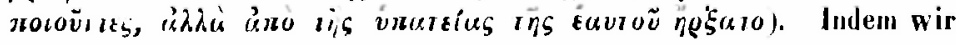


somit der von Heeren, Weizsäcker nnd Thouret vertretenen ansicht, dass Cicero die letzte quelle der iibercinstimmenden albschnitte des Dio nnd Plutarch gewesen, uns anschliessen, habeu wir noch die frage zu beantworten. ob den beiden schiriftstellern Cicero's Memoiren selbst vorgelegen haben oder durch die vermittlung einer ahgeleiteten quelle ihnen zug̈̈nglich geworden sind.

Was für die letztere anmalime hisher anfgefülirt wurde, beruht auf ziemlich vangen vermuthungen, wie z. b.. dass Dio und Plutarch mit dem lobe Cicers's sparsimer gewesen seien. als sich dies von der selbstialberhehung (icero's voraussetzen lasse (Peter a. a. o. 1. 133), dass Dio's unrichtige angabe von der ausweisung des Cutitina (XXXVII, 33,1) anch bei Livius geslanden habe (Grasshof a. a. o. p. 16. cpit. I. Cll. Catilina urbe pulso. Vgl. auch Eutrop. II, 15 a Cicerone ubo expulsus est), dass die von Dio und Plutarch gegen Caesar, Crassus und Antonius gemachten ausfalle nicht von dereu zeitgenossen Cicero herrühren kïnnten (Besser a. a. o. p. 46. 47 etc.). (irïssere beweiskralft kommt dem umstande zu, dass Dio die ereiguisse vor und nach der catilinarischen verschwörung wahrscheinlich ans Livius geschöpft, dass dieser die politische hedeutung des Cicero ganz besonders betont (ep. I. CIl ea coniuralio indus!ria M. Tulli Ciceronis erula esl) und die geschichte der verschwörung im einklang mit der von Dio und Plutarch gegebenen version geschildert hat. Besonders bedeutsam ist die uibereinstimmung zwischen den von Julius Obsernuens, dem epitomator des Livius iiberlieferten prodigien des jahres 68!) einerseits und den correspondirenden angahen des Dio Cassius XXXVII, 9 und 34 und Cicero Cat. III, 8 und 9) andererseits. Bindlich enthalt nicht nur die darstellung des persïnlich gegen Cicero eingenommenen Dio, sondern auch des Plutarch, und zwar an mehreren mit einander correspondirenden stellen invectiven gegen Cicero, die mïglicherweise beide schon in ihrer vorlage gefunden hiben (vgl.

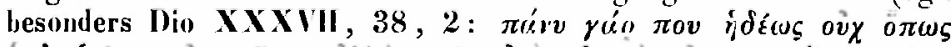

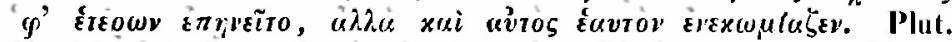

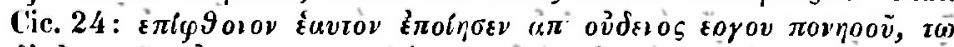

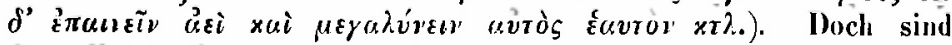
dies alles, wie gesigt, nur vermuthungen, und muss der beweis, dass die darstellung des Livius, ebenso wie die des Dio und Plutarch, auf Cicero's aufzeichnungen beruhte, erst moch erbracht werden.

Auch die erledigung der frage nach der beuutzung des Sallustius bereitet manche schwierigkeit, um so mehr, als Appian, der ja seinerseits ebenfalls dem Sillust und wahrscheinlich iuch der quelle des Dio-Plutarch gefolgt ist, lier zugleich mit in betracht kommt. So viel ist gewiss, dass Sallust mur an sehr wenigen stellen von Dio eingesehen und auch da nur subsidiir verwendel worden ist. Am ersten kümmen wir noch Sallust's anspruch auf cal!. 30 
des Dio, wo es sich um die von Tarpuinius gegen Crassus vorgebrachte denunciation landelt (Sall. Cait. 48), gelten lassen, während in anderen stellen in erwigung gezogen werden muss, dass auch der "originalschriftsteller" Sillust die von ilım berichteten thatsachen zum grossen theile aus schriftquellen geschöpft und sich namentlich an Cicero auf ua. engste angeschlossen hat.

Mit der erklärung der bei llio sich findenden anklänge an Appian (Wiedemann, Philologus XXII, p. 498) wird uns aber ein noch schwierigeres problem zu lïsen aufgegeben. Vergegenwärtigt man sich, dass eine benuzung des Appian an anderen theilen des dionischen werkes nicht nachgewiesen werden kann, ferner dass Dio, im besitze des livius, ferner der reichhaltigen quelle des Plutarch und des sallustianischen Catilinit, kaum eine veraulassung zu weiteren quellenstudion haben konnte, so wird man sich kium entschliessen kïnnen, jene allerdings aullallende ibereinstimmung ans einer abhängigkeit des Dio von Appian zı erklären. Doch setzen wir die hauptstellen lieher :

\section{Dio $\times \times \times 111,4$}

Appian, Bell. civ. II, 2

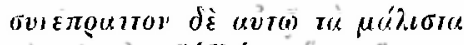
ir $\mu$ हो हो' xiei ó Ávtoulos ó llovithos

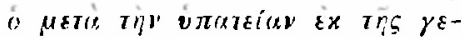

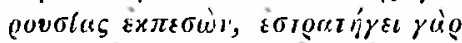

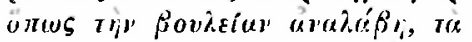

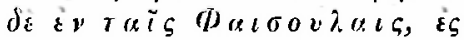

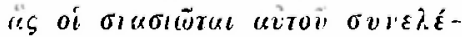

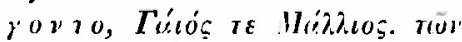

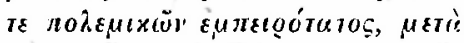

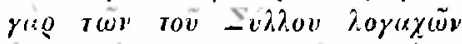

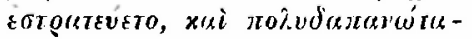

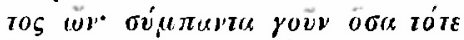

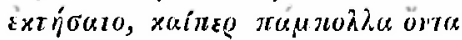

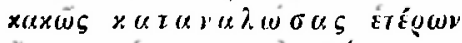
๕g แน́n

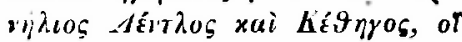

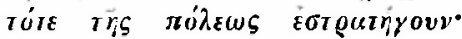
(x)

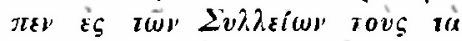

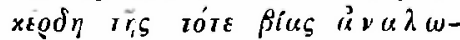

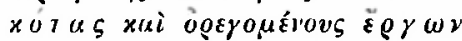
o $\mu$ of $\omega \nu, \varepsilon \varsigma \mu \varepsilon y$ (T) $\alpha+\sigma o v-$

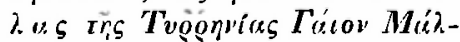

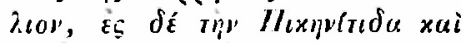

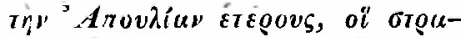

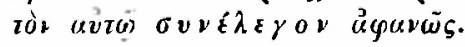

Die sämmtlichen zusäize Dio's zu den angaben des Appian sind durch Plutarch und Cicero beglaubigt; den Manlius nennt auch Cicero (at. II, 6, 14 als cenlurio und charakterisirt ilın an anderer stelle als exercilulione robustum und als einen von den Sullanern, qui se in insporalis ac repentinis pecuniis sumptuosius insolentiusque iactamml. . . qui atian nomunllos ayresles homines. . . in eamdem illum spom rapinarum velorum impulermit (Cic. (at. II, ), 20). Aelinlich hal sich Sallust Cat. 16. 28 ansugitriakt. Wenn es ferner hei Plutarch (ive. I/ von deu 


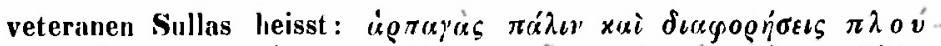

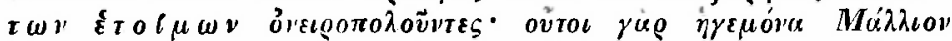

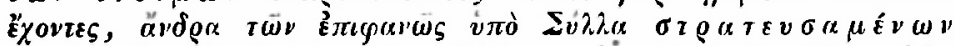
- so wird mit diesen stellen Wiedemann's ansicht allerdings nicht völlig widerlegt, wohl aber wenigstens auf die möglichkeit einer anderen, als der von ilım vorgeschlagenen erklärung der ibbereinstimmung des Dio mit Appiau hingewiesen.

Diodor (XIL, $5^{\text {a }}$ ed. Dindorf) kann als Quelle des Dio Cassius nicht ernstlich in betracht kommen, da von allem anderen ab-

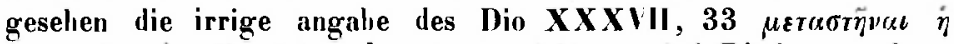

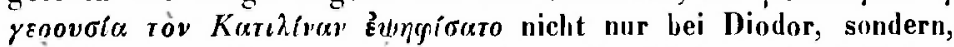
wie oben bemerkt: auch in der epitome des Livius und hei Eutro-

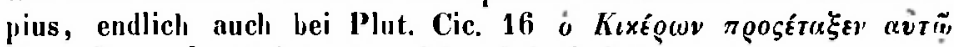

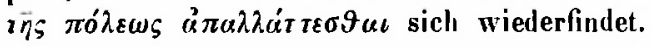

\section{Die kriege des Caesar gegen die Gallier (Lib. XXXVIII,} 1-50. XXXIX, 1-5 und 40-53. XL, 1-11 a. $31-43$ ).

Für die in den übrigen kapiteln des 37. buches, sowie in den beiden folgenden büchern enthaltene erziilılung der städtischen ereignisse wälırend der jalıre 692-700 liegen fast gar keine bestimmten anhaltspunkte vor, durch die ein schluss auf Dio's quellen gestattet wäre. Es muss also dalingestellt bleiben, ob Dio Cassius, wie Grasshof (nr. 3 p. 17-28) annimmt, auch hier dem Livius gefolgt ist. Vermittels eines weiten unweges gelangt Grasshof (a. a. o.) dazu, auch die nachrichten Dio's über die gallischen feldzüge des Caesar auf Livius zurückzufülıren. Indem er nämlich von der überraschenden ähnlichkeit von Caesar s Commentarien mit den entsprechenden angaben des Dio ausgelit, glaubt er bei dem letzteren eine reihe von abweichungen, zusätzen und berichtigungen zu finden, die nur der feder eines mit den verlältnissen aufs genaueste vertrauten zeitgenossen entstammen können, der höchstwalırscheinlich in der näclısten ungebung Caesars und augenzenge der besprochenen ereignisse gewesen sei. Durch Dio's angaben werden manche ïbertreibungen Caesars, besonders aber solche stellen, an denen dieser seine misserfolge zu vertuschen suchte, berichitigt, wenn dabei auch im ganzen sine ira et studio verfaliren worden ist. Im folgenden schliesst sich nun Grasshof an die von Evssenlardt (nr. 2) ïber die glaubwürdigkeit von Caesars Commentarien gefülirten untersuchungen an, welche die erklärung für jene von Caesar abweichenden züge der dionischen darstellung in folgender stelle des Sueton (Caes. 56) gesucht haben: Pollio Asinius parum diligenter parumpue integra veritate composilos [Caesaris commentarios] pulal qumm Caesar pleraque et quae per alios erant gesta, temere crediderit, et quae per se. vel consulto vel etiam memoria lapsus, perperam ediderit, existi- 
matque rescriplurum ot correcturum fuisse. Wihrend sich nun Eyssenlardt mit der vermutlung begnügt, dass Dio neben den Commentarien des Caesar noclı „andere unverächtliche quellen“, also entweder direkt oder indirekt das geschichtswerk des Asinius Pollio benutzte, geht Grasshof noch einen schritt weiter: da er es für ausgemacht hält, dass Dio nirgends an der ilım vorliegenden quelle kritik geübt oder dieselbe durch eine zweite vorlage zu ergänzen suchte, andererseits die direkte benutzung des Caesar durch die festgestellten abweichungen und widersprüche ausgeschlossen ist, endlich auch Asinius Pollio unmöglich die Commentarien des Caesar benutzt oder gar ausgesclirieben haben kann .-. aus allen diesen gründen bleibt kein anderer ausweg offen, als dass Dio einem gewährsmann gefolgt ist, der die darstellung des Caesar mit der des Asinius Pollio combinirt hatte. Wer dieser autor gewesen ist, darnach brauchen wir nicht lange zu fragen: auch hier ist Livius des Dio quelle gewesen, auod omnis operis Liviani forma, Dionis operi congrua, ei arridere debebat. Em unser urtheil kurz zusammenzufassen, so halten wir die beiden ersten vordersätze für falsch, den dritten für unerwiesen, das resultat für ein äusserst fragwürdiges. Was vor allem die für Dio Cassius angenommene methode der quellenbenuzung anlangt, so haben wir ihn im vorausgehenden zu wiederholten malen die berichte melirerer vorlagen verbinden sehen. In der vorrede versichert er uns, dass er das gesammte vorhandene

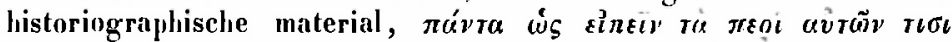

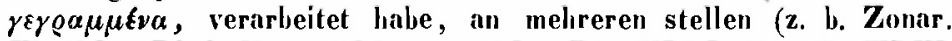
VI, 26. Dind. II, p. 166, 13-16. Dio XIIII, 2's, 2. XIIII, 26, 2 etc.) erklärt er, widersprüche in den ihm vorliegenden quellen zu finden; dass er es namentlich liebte. durch die lieranziehung von monographieen die angaben seiner annalistischen quellen zu ergänzen, geht aus der benutzung des anonymus (Coelius?) für die geschichte des zweiten punischen krieges, ferner des Catilina und der Historien des Sallust klar genug hervor. Was konnte also Dio näher liegen, als die berülımten Commentarien Caesars seiner darstellung der Gallierkriege zu grunde zu legen? Aber jene widersprüche, zusätze und berichtigungen? Suchen wir uns, über ilıren ursprung durch die betrachtung einzelner fälle klarbeit zu verschatfen! Von Eyssenliardt (nr. 2, p. 759), wie von Grasshof ist darauf hingewiesen worden, dass Dio XXXVIII, 31 den grund für die auswanderung der Helvetier ganz richtig in der übervölkerung ilires gebirgslandes erblicke, während Caesar angiebt, sie seien dazu von dem herrschsüchtigen Orgetorix verleitet worden. Dio füge aber sogar noch bei, dass Caesar selbst mit ehrgeizigen plänen in die provinz gekommen sei und den ausbruch von unruhen lebliaft gewünscht habe; dem Dio zufolge führt ferner Orgetorix die Helvetier aus ilırem gebiete, wälırend er nach Caesar schon vor dem auszuge seinen tod fand. Diss die letztere angabe Dio's 
falsch ist, hat auch Eysseuhardt zugegeben und kann also sie wenigstens nicht jener "vortrefflichen" quelle entstammen. Hahen wir aber die wahl, den grohen schnitzer entweder dem Dio Cassius oder seiner angeblichen quelle Livius zuzuschreiben, so kann die antwort nicht zweifelhaft sein; das sündenregister Mio's ist schon nich den untersuchungen ron Fabricius, Reimarus und Wilmans ein so reichhaltiges, dass jenes ans flüchtiger lecture seiner vorlage hervorgegangene versehen des Dio dagegen gar nicht in betracht kommt. Hinsichtlich der ursachen jener answanderung aber kinn ich keinen wesentlichen widerspruch zwischen Dio und Caesar entdecken, der, nachdem er der untriebe des Orgetorix erwähnung gethan, ansfülırlich die unzufriedenheit der Helretier schildert und mit den worten schliesst (Bell, Gall. I, 2, 5): pro multitudiue autem hominum et pro gloria belli atque fortitudinis angustos se

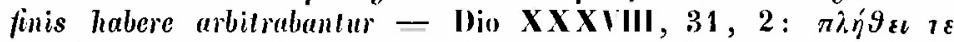

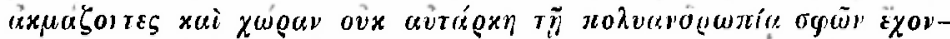

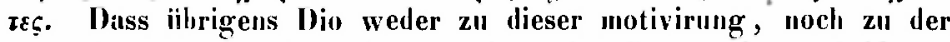
hervorbebung des elırgeizigen strehens des Caesar einer schrifistellerischen quelle bedurfte, wird jeder, der ohne voreingenommenheit anch nur ein einziges buch des Dio durchgelesen bat, uns zugeben müssen. Räsonniren und pragmatisiren um jeden preis - ist seine losung; er liest in den herzen der menschen und kommt ihren geheimsten gedanken anf die spur; politische ereignisse ohne den hintergrund des kampfes und einflusses persönlicher interessen sind dem Dio ein unding, und am schlusse eines seiner zithlreichen didaktischen excurse ïber den übergang der republik zur monarchie bebt er es als einen besonderen vorzug seines geschichtswerkes hervor, dass in ihm die wechselbezichungen zwischen dem geistesleben der handelnden personen und den grossen ereignissen der weltgeschichte zur darstellung kommen (XINI, 35). Diese neignng IDio's zu rationalistischer klügelei liefert uns nun iuch den schliussel zu der erklïrung einer reihe jener von Caesar's Commentaricn ahweichenden angaben, in denen Grasshof correcturen des Asinius Pollio entdeckt hat. So giebt z. b. Dio XXXıX, 48,5 als grund für den eiligen rückzug des Caesar über den Rhein in jahre 699 den umstand an, dass die Sigambern sich nach unzugänglichen orten geflïchtet, die Sueven dagegen sich zu ihrem schutze bereit gezeigt hätten; die zweite expedition ins innere von Deutschland hat Caesar nach Dio XI, 32, 2 aus furcht vor den Sueven aufgegeben. Mit der ersten stelle steht Caesar IV, 18. 19 üherhaupt gar nicht in widerspruch, da auch bei ihm die Sueven sich zu einer entscheidungsschlacht rüsten. An der zweiten stelle ist allerdings Caesar's darstellung (VI, 10. 29) eine wesentlich verschiedene; er will den rückzug angetreten haben, weil die Sueven mit allen ilıren bundesgrenossen sich in den äussersten winkel ihres gelietes zurückgezogen hätten, "um dort die in- 
kunft des rimischen heeres z" erwarten. Zu welchem anderen resultate aber hat Dio, wenn er nach einer plausibeln erklärung von Ciesar's unerwartetem entschlusse fragle und dahei wie immer seinem nüchternen, pessimistischen urtheil folgte, gelangen kïnnen, als dass Citesar mit dem deutschen anzubinden, keine "lust hatte" (Mommsen, Rïm. gesch. ld. $3^{4}$, 1. 255). Zu lieser an Caesar's herichte geülten kritik hat Dio, ebensowenig wie die in diesem punkle mit ilım ïbereinstimmenden modernen historiker einer bericlitigenden nebenquelle bedurft. Aus Livius aber konnte Dio, was Grasshof ganz entgangen ist, jene angabe am allerwenigsten geschöpft haben, da auch bei diesem (epit. I. CVII) C'aesir, „weil er keinen feind in Jeutschland vorgefunden hatte" nach (iallien zurïckkehrt. - Besonders gravirend sind nach Grasshof die albweichungen des Jio XI, 8-10 von C'aesar's erzählung der belagerung des legaten $(2$. Cicero durch die Nervier (V, 42--51). Es sind folgende: Nachdem Caesar durch einen sklaven eines dem (icero treu gebliebenen Nerviers die bedringniss seines legaten erfaliren hatte (Cates. BG. V, 45), lasst er den Cicero durch einen gallischen reiter von seinem herannahen verständigen (Caes. B(i. V. 48), weil er jenem sklaven als einem landsmanneder belagerer kein vertrauen sehenkte; er benutzt zum marsche die nachte, um während des tages an verborgenen orten zu rasten (!); die Nervier erfahren seine ankunft desshall nicht durch kundschafter, sondern erst durch die freudigen mienen der belagerten. Sollte diesen abentenerlichen ausschmückungen des von Ciaesar geschilderten sichverlailtes in der that der bericht eines augenzengen zu grunde liegen! Oder wird uns nicht auch hier, wie an einer anderen ebenfalls von einer nächtlichen expedition des Caesar erzählenden stelle (Jio XI, 35, 4 $4=$ Caes. BG. VI, 35, 7 , vielmehr ein weiterer heweis dafür geliefert, dass Dio wie bei seinen zahlreichen, von anfang bis zu ende erfundenen reden, so auch bezüglich seiner detailschilderungen in erster linie von rhetorischen rücksichten ausgegangen ist? Wir müssen es uns versagen, die sämmtlichen von (irasshof angeführten stellen des Dio hier zu besprechen; es sei nur so viel gesagt, dass, von zwei stellen alugesehen, die sämmllichen zusätze des IVio Cassius nichts als armselige variationen und erweiterungen der Commentarien des Caesar enthalten, die ohne allen zweifel seine einzige quelle gewesen sind. Sollte dennoch die vermittlung des Livius angenommen werden müssen, für die bis jetzt wenigstens noclı kein einziges argument beigebracht worden ist, so wïrde natïrlich das oben ausgesprochene urtheil auch auf ilın auszudehnen sein. (Aegen eine benutzung des Livius spricht übrigens besonders der umstand, dass Dio die darstellung der ereignisse während der jahre $692-700$ in ganz anderer weise, wie Iivius angeordnet hal, wie sich aus folgender gegeniiherstellung ergielit: 
Liv. epit. I. CIII-CVIII.

IIio.

1. Krieg gegen die Helvetier.

1. Triumpli des Pompejus.

2. Krieg gegeu Ariovist.

3. Verbanung des Cicero anfgehoben.

4. Krieg gegen die Belgen, Ambianen, Suessioneu etc.

5. Krieg gegen die Nervier etc.

6. Verhandlungen über Cyperu.

7. Verhandlungenüber Aegypten.

8. Krieg gegen die Velleter etc.

9. Stadtische angelegenheitendes jahres 698 und 699.

10. Krieg gegeu die Tencterer und Usipeter.

11. Erster Rheinübergang.

12. Erste und zweite expedition nach Britannien.

13. Tod der Julia.

\section{Empörung des Ambiorix.}

15. Feldzug gegen die Parther.

16. Krieg gegen die Trevirer etc.

17. Ermordung des Clodius etc.

18. Krieg des Vercingetorix.
1. Krieg gegen die Helvetier.

2. Krieg init Ariovist.

3. Krieg gegen die Belgen.

4. Krieg gegen die Nervier etc.

5. Verbannung des Cicero aufgehoben.

6. Verhandlungen über Aegypten.

7. Verhandlungen über Cypera.

8. Städt ische angelegenheiten des jalires 698 und 699 .

9. Krieg gegen die Veueter etc. 10. Krieg gegen die Tencterer und Usipeter.

11. Erster Rheinübergang.

12. Erste expedition nach Britannien.

13. Stadtische angelegenleiten tod der Julia.

13. Zweite expedition nach Britannien.

14. Empörung des Ambiorix.

15. Feldzug gegen die Parther. 16. Krieg gegen die Trevirer etc. 17. Krieg des Vercingetorix.

18. Ermordung des Clodius etc.

Ist es auch nicht von vornherein unmöglich, dass Dio aus seiner aunalistischen quelle die auf die Gallierkriege bezüglichen abschnitte aushob und nach eigenen gutdünken zusammenstellte, so halten wir es doch für weit wahrscheinlicher, dass jene verschiedenheit der anordunng ans der gleichzeitigen beuntzung des Livius und Caesar hervorgegangen ist. Dafür spricht namentlich der umstand, dass Dio im gegensatz zu Iivius an die erste britannische expedition Caesars, mit deren geschichte das vierte buch der Commentarien abschliesst, die schilderung der städtischen ereiguisse des jalıres 699 anknüpft und dann ebenso wie Caesar mit der darstellung des zweiten britannischen krieges ein neues buch beginnen lasst. Also wird anch nach dieser seite unsere annahme einer direkten benutzung der Commentarien des Caesar durch Dio Cassius bestätigt.

Thouret (nr. 8, p. 330), der mit recht darauf hingewiesen hat, dass fast alles, was uns bei Orosius, Florus, Eulropius, Plutarch, Appian, Sueton und Dio Cassius über die gallischen feld- 
züge des Caesar überliefert wird, in letzter linie auf dessen Commentarieu als einzige quelle zurückgeht, nimmt von diesem urtheil nur einen einzigen alsschnitt des Dio Cassius aus, die darstellung der gegen Ariovist bei Mühllhausen gelieferten scblacht: s. Dio XXXVIII, 49. 50. Was hier über die bewaffinung und kampfesweise der Germanen und den verlauf des von Caes. BG. I, 52. 53 ganz flüchtig geschilderten kampfes erzähılt wird, ist nach Tloouret wahırscheinlich aus einer monographie eingelegt worden, die sich ausschliesslich auf die schlacht bei Mühlhatusen beschränkte und, wie die schrift des Aurunculeius Cotta ibler die zweite expedition des Caesar nach Britannien, einen römischen offizier zum verfasser hatte. Alles andere aber, was bei Dio jener episode folgt und vorausgeht, beruht auf Caesar's eigenem berichte; eine benutzung des Asinius Pollio durch Dio oder dessen quelle ist nach Thouret schon um desswillen ausgeschlossen, weil jener eine ausfülırliche darstellung der gallischen feldzüge Caesars überhaupt nicht verfasst, sondern derselben nur gelegentlich erwälınung gethan hat. Auch das ist freilich nur eine vermuthung; eine ausgemachte thatsache aber ist es, dass in der uns über die Gallierkriege erhaltenen tradition sich spuren einer dem Caesar feindlich gegenüberstehenden oder auch nur von ihm unabliängigen quelle sich nicht nachweisen lassen. Und ich trage kein bedenken, diese ansicht auch auf den in rede stehenden bericht Dio's über die niederłage des Ariovist auszudehnen. Die bei Caesar fehlenden wichtigeren angaben Dio's sind folgende:

1) In folge des raschen ansturms der Römer vermögen die Germanen weder ihre langen schlachtschwerter, noch ihre $\beta$ ga $v^{\prime}-$

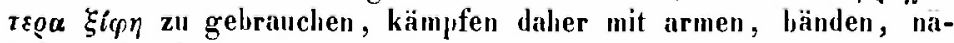
geln und zälınen.

2) Nachdem der sieg lange unentschieden bin- und hergescliwankt hat, verleilst die ïlerlegenheit der kurzen, spitzigen schwerter der Römer über die langen schwerter der Deutschen den ersteren die oberband.

3) Die Germanen treten nun in kreise von durchschnittlich 300 mann zusammen und stehen so enge gedrängt, dass sie sich nicht rühren können.

4) Vor dem angriffe auf diese phalangen (Caes. I, 52, 4) werfen die Römer speere und schilde als unnütz von sich.

5) Manche der unter diesen schlachithaufen getödteten Deut-

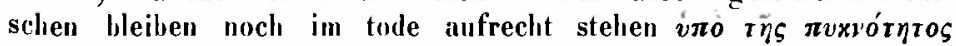

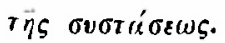

Dem ersten und letzten zusatze historische wahrheit zuzuschreiben, seheint sehr bedenklich; namentlich wird durch den ersten zusatz die bemerkung Caesar's, dass fïr das abschleudern der wurfspeere kein raum übrig blieb, in monströser weise gesteigert. bie in dem zweiten zusalze betonte überlegenheit der römischeu 
bewaffinung vor der der Gallier (Dio XXXVIII, 49, 4: $\sigma_{\mu l-}$

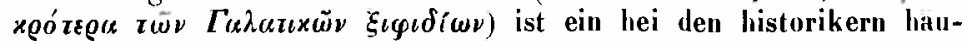
fig vorkommender gemeinplatz. Der dritte zusatz enthält eine vielleicht mit, vielleicht aber auch ohne sachkenntniss gegebene erklärung von Caesar's worten : at Germani celeriter ex consueludine sua phalange facla impetus gladiorum exceperunt. Durch den vierten zusatz endlich wird die augabe Caesar's, dass einzelne römische soldaten mit ihren händen (die sie also frei haben mussten) den Deutschen ihre schilde entrissen, einfach in die länge gezogen. ob die poetische ausmalung von Caesar's lakonischer darstellung auch hier Dio's eigenstes werk ist, kanu mit sicherheit nicht entschieden werden. Die worte des Orosius VI, 7: Pugna maxime gravis ex phalange Germanorum fuil, quam coacto in unum agmine scutisque supra capila conlexlis ad inrumpendam Romanorum aciem luti undique praestruxerant - enthalten eine ähnliche umschreibung der oben angeführten worte Caesar's, wie der zweite zusatz des Dio und könnten also auf Livius, als die gemeinsame quelle beider schilderungen hinweisen. Doch ist diese folgerung sehr ungewiss und auch für die beurtheilung des werthes jener zusätze von untergeordneter bedeutung, da in jedem falle der abenteuerlichen schlachtbeschreibung des Dio gegenüber das grösste misstrauen geboten ist. (Fortsetzung folgt.)

Wiirzburg.

Hermann Hatpl.

\section{Zu Tacit. Hist. I, 4.}

Nachdem Tacitus bei schilderung des innern zustandes der stadt Rom nach Nero's tode der patres und der equites illustres erwähnung gethan, fährt er tort: pars populi integra el magnis domibus adnexa, clientes liberlique damnatorum et exulum in spem erecti: plebs sordida et circo ac theatris sueta, simul deterrimi servorum aut qui adesis bonis per dedecus Neronis alebantur, maesli et rumorum avidi: hier ist, um richtig zu erklären, vor allem die genaue entsprechung der einzelnen glieder und die variation zu beachten: denn pars populi ist s. v. a. pars plebis, und plebs gleich pars plebis oder pars populi: dann bilden die worte clientes ... exulum nur eine nähere bestimmung, einen theil der pars populi .. adnera: hier lässt 'Tacitus das asyndeton zu, während im zweiten theil er simul an dieser stelle hat, so dass gar nichts vom asyndeton im zweiten theile sich findet: danach schon ergiebt sich, dass pars populi integra der plebs sordida entspricht, die grundlage für die richtige auffissung von integra, die übrigens schon Lipsius gegeben: der gute. unbescholtene theil der grossen masse, der bürgerlichen bevölkerung: Döderlein z. b. stellt dies integra irrthümlich dem adesis bonis entgegen.

Ernst von Leulsch. 